\title{
A Metric for Analyzing Effective On-Chip Inductive Coupling *
}

\author{
Guoan Zhong, Cheng-Kok Koh, and Kaushik Roy \\ School of Electrical and Computer Engineering \\ Purdue University, West Lafayette, IN 47907-1285 \\ \{zhongg,chengkok,kaushik\}@ecn.purdue.edu
}

\begin{abstract}
In this paper, we propose a metric for effective inductive coupling: the matrix $(R+j \omega L)^{-1}$, where $R$ and $L$ are the resistance and inductance matrices. We use this metric to analyze the effectiveness of shields on reducing inductive coupling. Our analysis shows how the resistances of shields affect the effective inductive coupling between signal nets. SPICE simulations are carried out to validate the proposed metric.
\end{abstract}

\section{INTRODUCTION}

Continued scaling of semiconductor technology has brought the issues of signal integrity to the forefront. Most existing studies focused on crosstalk due to capacitive coupling. At multi-gigahertz frequency range, the inductive effect cannot be ignored. This paper concentrates on the inductive coupling of on-chip interconnects.

It is known that the capacitive coupling is short-range. Within one layer, only the capacitive coupling from the nearest neighbors has to be considered. On the other hand, inductive coupling has a longer range effect. It is not enough to consider only the nearest neighbors for inductive coupling. For this reason, it is harder to analyze the inductive coupling for interconnect design. Moreover, controlling the inductive coupling is more difficult than controlling the capacitive coupling.

Quiet signals, especially the dedicated shields can reduce the effective inductive coupling to some extent. In high performance microprocessors, a great number of shields are inserted to control the coupled noises [4, 6]. In [7], $2 \times$ mutual inductance screening rules are proposed. The potential victims of an inductive aggressor are those nearest neighboring wires whose total width is equal to or less than twice the width of the aggressor.

Many of the existing studies that dealt with on-chip inductive effects focused on the modeling and extraction of on-chip

*This research was supported in part by SRC (99-TJ-689), NSF (CCR9984553), and a grant from Intel Corporation. interconnect inductance. In [9], loop inductance was calculated in terms of partial inductances defined for wire segments. The Partial Element Equivalent Circuit (PEEC) model was widely used to analyze on-chip inductance $[8,3]$. Unlike the capacitance matrix, the inductance matrix is dense. Analyzing inductive coupling with the inductance matrix directly is not practical. In [2], the locality property of $L^{-1}$, where $L$ is the inductance matrix, is demonstrated and is used for inductance simulation. $L^{-1}$ has properties similar to those of the capacitance matrix, and it facilitates the analysis of the inductance effects. However, the locality property of $L^{-1}$ does not adequately explain the long range effect of inductive coupling.

In this paper, we show that the resistances of the interconnects also play an important role in determining the long range effective inductive coupling. We propose a new metric for effective inductive coupling: the matrix $(R+j \omega L)^{-1}$, where $R$ and $L$ are the resistance and inductance matrices. Our analysis of this metric on shield structure shows how the resistances of shields play a crucial role in shielding the inductive coupling between signal nets.

\section{A Metric for Effective Inductive Coupling}

Consider two signals $i$ and $j$ in a bus. To quantify the inductive noise induced on signal $i$ because of the switching in signal $j$, it is not enough to consider only $L_{i j} \frac{d I_{j}}{d t}$, because the switching in aggressor $j$ also induces currents in the remaining nets (including the victim $i$ ). Those induced currents will also contribute to the noise in the victim $i$. For example, the induced currents in the shields (if present) may greatly cancel the noise in the victim. This is captured by the following equation:

$$
V_{i}=R_{i} I_{i}+\sum_{k} L_{i k} \frac{d I_{k}}{d t},
$$

where $R_{i}$ is the resistance of signal $i, L_{i k}$ is the partial mutual inductance between net $i$ and $k$ and $I_{k}$ is the current in net $k$. 
To obtain the inductive noise $V_{i}$, we have to know all the induced currents. This is captured by the following linear matrix equation:

$$
V=R I+L \frac{d I}{d t}
$$

where $V, I$ are the voltage and current vectors, respectively; $R$ and $L$ are the resistance and (partial) inductance matrices, respectively. Rewriting Eqn.( 2) in the frequency domain,

$$
V=(R+j \omega L) I
$$

For the analysis of how the resistances of wires affect effective inductive coupling, we rewrite Eqn. (3) as

$$
I=(R+j \omega L)^{-1} V,
$$

and define

$$
Y=G+j K=(R+j \omega L)^{-1} .
$$

We shall analyze matrix $Y$, or equivalently, matrices $G$ and $K$ for inductive coupling. The physical meaning of $Y_{i j}$ is the impact of the switching of the aggressor $j$ on the current in the victim $i$; the larger the magnitude, the stronger the inductive coupling.

To write $G$ and $K$ in terms of $R$ and $L$, we define

$$
€=\omega L \text {. }
$$

From Eqn. (5), we have

$$
(G+j K)(R+j €)=I .
$$

Solving the preceding equation gives

$$
K=-\left(R £^{-1} R+€\right)^{-1}
$$

and

$$
G=-K R £^{-1}
$$

Note that matrix $K$ defined in this paper is different from the " $K$ " matrix in $[2,1]$, where " $K$ " is defined as $L^{-1}$. Here, both $G$ and $K$ are some combinations of $R$ and $L$. [2] demonstrates the locality of $L^{-1}$ and uses $L^{-1}$ in the simulation. By itself, however, $L^{-1}$ is not a good metric for effective inductive coupling, because it does not consider the effect of resistances. In this study, we combine $L^{-1}$ and resistance $R$, and propose a new metric for effective inductive coupling: $(R+j \omega L)^{-1}$.

In the following section, we shall use this metric to study the effectiveness of the shields on reducing the effective inductive coupling.

\section{ANALYSIS EXAMPLES}

It is known that low resistance shields is preferred to reduce effective inductive coupling. The smaller the shield resistances, the better the shielding effect. In this section we use the proposed $(R+j \omega L)^{-1}$ to explain why low resistance shields work better in bus structures. The locality of $L^{-1}$ [2] is utilized in this analysis.

\section{A. Theoretical Analysis}

We begin with a simple structure with only two signals separated by one shield. For clarity of presentation, we ignore the skin effect and proximity effect. The impedance matrix of the structure is

$$
Z=\left[\begin{array}{ccc}
R_{s}+j \omega L_{s} & j \omega M_{s g} & j \omega M_{s s} \\
j \omega M_{s g} & R_{g}+j \omega L_{g} & j \omega M_{s g} \\
j \omega M_{s s} & j \omega M_{s g} & R_{s}+j \omega L_{s}
\end{array}\right]
$$

where $R_{s}$ and $L_{s}$ are respectively the resistance and (partial) self inductance of the signal wires; $R_{g}$ and $L_{g}$ are respectively the resistance and (partial) self inductance of the shield; $M_{s g}$ and $M_{s S}$ are respectively the (partial) mutual inductance between a signal wire and the shield, and that between the two signals. We have,

$$
Y_{13}=Y_{31}=\left(\omega^{2}\left(L_{g} M_{s s}-M_{s g}^{2}\right)-j \omega M_{s s} R_{g}\right) /|Z|,
$$

and

$$
L_{13}=L_{31}=\left(M_{s g}^{2}-L_{g} M_{s s}\right) /|L| .
$$

If $L^{-1}$ is tridiagonal, we have $L_{13}=L_{31}=0$. Thus,

$$
L_{g} M_{s s}-M_{s g}^{2}=0
$$

If we have a perfect shield, i.e., $R_{g}=0$, then

$$
Y_{13}=Y_{31}=0
$$

Therefore, there will be no coupling between the two signals. However, if we have perfect signal wires, i.e., $R_{s}=0$, but a resistive shield, i.e., $R_{g} \neq 0$, the two signals will be coupled.

Now, we shall extend our analysis to consider a general shielded structure.

Observation 1 if $L^{-1}$ is tridiagonal, and two signals are separated by one perfect shield, there will be no effective inductive coupling between these two signals.

First, we consider the fully shielded structures, in which a $\mathrm{P} / \mathrm{G}$ shield is inserted between every pair of neighboring signals as shown in Figure 1. This is also known as the dense 


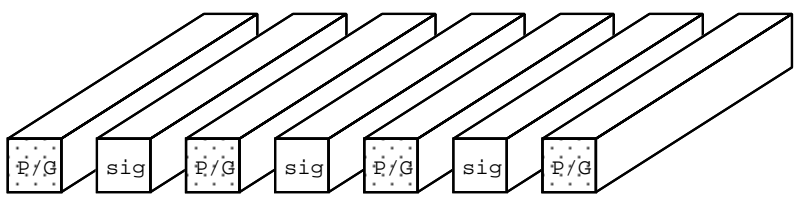

Fig. 1. A fully shielded structure with seven wires: three signal nets and four shields $(\mathrm{P} / \mathrm{G})$.

wiring fabric proposed in [5]. We assume that the even wires are shields and the odd wires are signal nets. We define:

$$
\zeta=\longleftarrow^{-1}
$$

Since $L^{-1}$ (and hence, $\iota^{-1}$ ) has locality, for simplicity, we assume that $\zeta$ is a tridiagonal matrix.

We can rewrite Eqn. (8) as:

$$
\begin{aligned}
K & =-\left(R \zeta R+\zeta^{-1}\right)^{-1} \\
& =-\zeta(R \zeta R \zeta+I)^{-1}
\end{aligned}
$$

Suppose the shields are perfect, i.e., they have no resistances, then $R$ is diagonal and the even entries in the diagonal are zeros:

$$
R=\left[\begin{array}{cccccc}
R_{1} & & & & & \\
& 0 & & & & \\
& & R_{3} & & & \\
& & & 0 & & \\
& & & & \ddots & \\
& & & & & R_{n}
\end{array}\right] .
$$

As $\zeta$ is tridiagonal, $R \zeta$ is tridiagonal with zero even rows. Hence, $R \zeta R \zeta=(R \zeta)^{2}$ has the same format:

$$
R \zeta R \zeta=\left[\begin{array}{cccccc}
\star & \star & & & & \\
0 & 0 & 0 & & & \\
& \star & \star & \star & & \\
& & 0 & 0 & 0 & \\
& & & & \ddots & \\
& & & & \star & \star
\end{array}\right],
$$

where each $\star$ denotes a non-zero entry. Hence,

$$
R \zeta R \zeta+I=\left[\begin{array}{cccccc}
\star & \star & & & & \\
& 1 & & & & \\
& \star & \star & \star & & \\
& & 1 & & \\
& & & \ddots & \\
& & & \star & \star
\end{array}\right] .
$$

It is straightforward to prove that $(R \zeta R \zeta+I)^{-1}$ has the same format as $R \zeta R \zeta+I$ :

$$
(R \zeta R \zeta+I)^{-1}=\left[\begin{array}{cccccc}
\star & \star & & & & \\
& 1 & & & & \\
& \star & \star & \star & & \\
& & & 1 & & \\
& & & \ddots & \\
& & & \star & \star
\end{array}\right] .
$$

Now, from Eqn 15,

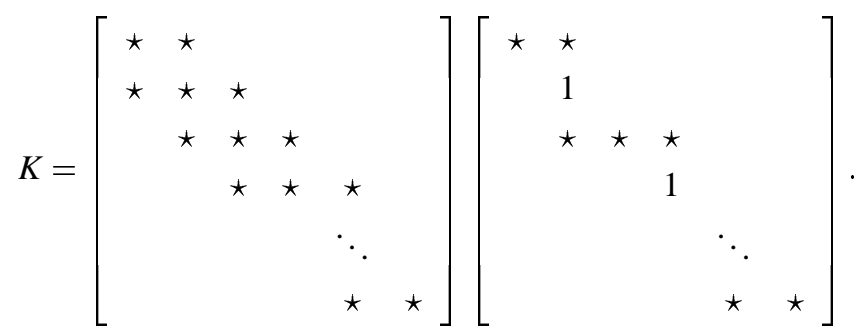

It is trivial to verify that for odd $i, j$ and $i \neq j$ :

$$
K_{i j}=0, G_{i j}=0 .
$$

Thus, there is no effective inductive coupling between any pair of signals. (Similar conclusion can be reached if the odd wires are shields.) However, the remaining entries of $K$ and $G$ may be quite significant. That implies that the coupling between a signal net and a shield (even if they are not adjacent) or the coupling between two shields can be quite strong. However, that should not be of concern as the voltage of shield is almost fixed all the time. It also implies that if the perfect wires are signal nets instead of shields, the signal nets would still be strongly coupled. It is also important to note that we cannot achieve effective inductive shielding by increasing the separations between wires alone, as that does not affect the $R$ matrix.

For a more general structure that has several signals between two shields (as illustrated in Figure 2), we can also prove, using similar steps, that $G_{i j}$ and $K_{i j}$ are both zero if two signals $i$ and $j$ are separated by at least one shield.

In general, if the $L^{-1}$ is not tridiagonal but has a bandwidth 


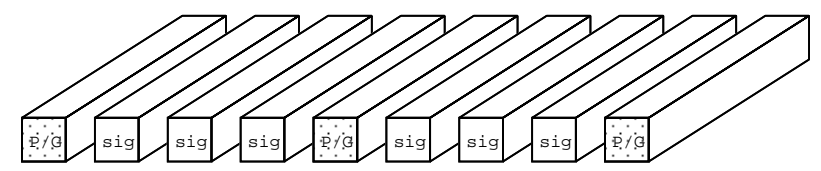

Fig. 2. A shielded structure with nine wires: three shields $(P / G)$ nets and six signals.

of $2 b+1$, we have the following observation:

Observation 2 if the bandwidth of the $L^{-1}$ is $2 b+1$, and two signals are separated by $b$ perfect shields, there will be no effective inductive coupling between these two signals.

\section{B. Numerical Analysis}

The conclusion that we can drawn from the preceding analysis is that perfect shields can provide very good inductive shielding. For the current technology, however, perfect shields do not exist. Thus, a common approach is to use wide shields to reduce the shield resistance. Although the shields are not perfect and $L^{-1}$ is not strictly banded, we can still perform a similar analysis, albeit a more elaborate one, to show that effective inductive coupling can still be significantly reduced by using wider shields. We skip the details of the more general analysis in this paper, and instead present some numerical results to justify the claim. Skin effects and proximity effects are considered in these numerical analysis.

Consider a fully shielded bus structure with seven parallel uniform wires (Figure 1). The cross section and length of each wire are $0.5 \mu \mathrm{m} \times 1 \mu \mathrm{m}$ and $100 \mu \mathrm{m}$, respectively. The separation between adjacent wires is $0.5 \mu \mathrm{m}$. The cross sections of the wires are meshed in order to capture the skin effect and proximity effect. At a frequency of $1 \mathrm{GHz}$,

$$
\begin{aligned}
& G=2.7 e-01 \times \\
& {\left[\begin{array}{rrrrrrr}
1.00 & -0.08 & -0.07 & -0.07 & -0.07 & -0.06 & -0.06 \\
-0.08 & \mathbf{0 . 9 9} & -0.08 & -\mathbf{0 . 0 8} & -0.07 & -\mathbf{0 . 0 7} & -0.06 \\
-0.07 & -0.08 & 0.99 & -0.08 & -0.08 & -0.07 & -0.07 \\
-0.07 & -\mathbf{0 . 0 8} & -0.08 & \mathbf{0 . 9 9} & -0.08 & -\mathbf{0 . 0 8} & -0.07 \\
-0.07 & -0.07 & -0.08 & -0.08 & 0.99 & -0.08 & -0.07 \\
-0.06 & -\mathbf{0 . 0 7} & -0.07 & \mathbf{- 0 . 0 8} & -0.08 & \mathbf{0 . 9 9} & -0.08 \\
-0.06 & -0.06 & -0.07 & -0.07 & -0.07 & -0.08 & 1.00
\end{array}\right],} \\
& K=-4.0 e-02 \times \\
& {\left[\begin{array}{lllllll}
1.00 & 0.67 & 0.49 & 0.38 & 0.32 & 0.28 & 0.27 \\
0.67 & \mathbf{0 . 9 4} & 0.62 & \mathbf{0 . 4 5} & 0.36 & \mathbf{0 . 3 1} & 0.28 \\
0.49 & 0.62 & 0.91 & 0.60 & 0.44 & 0.36 & 0.32 \\
0.38 & \mathbf{0 . 4 5} & 0.60 & \mathbf{0 . 8 9} & 0.60 & \mathbf{0 . 4 5} & 0.38 \\
0.32 & 0.36 & 0.44 & 0.60 & 0.91 & 0.62 & 0.49 \\
0.28 & \mathbf{0 . 3 1} & 0.36 & \mathbf{0 . 4 5} & 0.62 & \mathbf{0 . 9 4} & 0.67 \\
0.27 & 0.28 & 0.32 & 0.38 & 0.49 & 0.67 & 1.00
\end{array}\right] .}
\end{aligned}
$$

In the above two matrices, the odd columns and rows are related to shields. The remaining entries, which are in boldface, capture the coupling among signals. We can see that the coupling between the signals is significant. Now, we increase the the widths of those shields to $1.5 \mu \mathrm{m}$. We have

$$
\begin{aligned}
G= & 7.01 e-01 \times \\
& {\left[\begin{array}{rrrrrrr}
1.00 & -0.07 & -0.20 & -0.06 & -0.16 & -0.05 & -0.13 \\
-0.07 & \mathbf{0 . 3 9} & -0.07 & \mathbf{- 0 . 0 2} & -0.06 & \mathbf{- 0 . 0 2} & -0.05 \\
-0.20 & -0.07 & 1.00 & -0.07 & -0.20 & -0.06 & -0.16 \\
-0.06 & -\mathbf{0 . 0 2} & -0.07 & \mathbf{0 . 3 9} & -0.07 & \mathbf{- 0 . 0 2} & -0.06 \\
-0.16 & -0.06 & -0.20 & -0.07 & 1.00 & -0.07 & -0.20 \\
-0.05 & \mathbf{- 0 . 0 2} & -0.06 & \mathbf{- 0 . 0 2} & -0.07 & \mathbf{0 . 3 9} & -0.07 \\
-0.13 & -0.05 & -0.16 & -0.06 & -0.20 & -0.07 & 1.00
\end{array}\right], } \\
K= & {\left[\begin{array}{rrrrrrrr}
1.00 & 0.19 & 0.26 & 0.04 & 0.04 & 0.01 & 0.01 \\
0.19 & \mathbf{0 . 1 2} & 0.16 & \mathbf{0 . 0 2} & 0.03 & \mathbf{0 . 0 0} & 0.01 \\
0.26 & 0.16 & 0.82 & 0.15 & 0.18 & 0.03 & 0.04 \\
0.04 & \mathbf{0 . 0 2} & 0.15 & \mathbf{0 . 1 2} & 0.15 & \mathbf{0 . 0 2} & 0.04 \\
0.04 & 0.03 & 0.18 & 0.15 & 0.82 & 0.16 & 0.26 \\
0.01 & \mathbf{0 . 0 0} & 0.03 & \mathbf{0 . 0 2} & 0.16 & \mathbf{0 . 1 2} & 0.19 \\
0.01 & 0.01 & 0.04 & 0.04 & 0.26 & 0.19 & 1.00
\end{array}\right] . }
\end{aligned}
$$

It is clear that the coupling between the signals is very weak. In other words, widening the shields can achieve effective inductive shielding. It is also evident that the coupling between signals and shields and between shields is still very strong.

Next, we consider a more general structure with three signals between two shields, as illustrated in Figure 2. We want to show that if the shields are properly sized, there will be minimal effective inductive coupling between signals separated by one or more shields. For shield width, signal width, wire separation, wire thickness, and wire length of $0.5 \mu \mathrm{m}, 0.5 \mu \mathrm{m}$, $0.5 \mu \mathrm{m}, 1.0 \mu \mathrm{m}$, and $100 \mu \mathrm{m}$, respectively, the $G$ and $K$ matrices at $1 \mathrm{GHz}$ are

$$
\begin{aligned}
& G=2.71 e-01 \times \\
& {\left[\begin{array}{rrrrrrrrr}
1.00 & -0.07 & -0.07 & -0.07 & -0.06 & -0.06 & -0.06 & -0.05 & -0.05 \\
-0.07 & 0.99 & -0.08 & -0.07 & -0.07 & \mathbf{- 0 . 0 6} & \mathbf{- 0 . 0 6} & \mathbf{- 0 . 0 6} & -0.05 \\
-0.07 & -0.08 & 0.99 & -0.08 & -0.07 & \mathbf{- 0 . 0 7} & \mathbf{- 0 . 0 7} & \mathbf{- 0 . 0 6} & -0.06 \\
-0.07 & -0.07 & -0.08 & 0.99 & -0.08 & \mathbf{- 0 . 0 7} & \mathbf{- 0 . 0 7} & \mathbf{- 0 . 0 6} & -0.06 \\
-0.06 & -0.07 & -0.07 & -0.08 & 0.99 & -0.08 & -0.07 & -0.07 & -0.06 \\
-0.06 & \mathbf{- 0 . 0 6} & \mathbf{- 0 . 0 7} & \mathbf{- 0 . 0 7} & -0.08 & 0.99 & -0.08 & -0.07 & -0.07 \\
-0.06 & \mathbf{- 0 . 0 6} & \mathbf{- 0 . 0 7} & \mathbf{- 0 . 0 7} & -0.07 & -0.08 & 0.99 & -0.08 & -0.07 \\
-0.05 & \mathbf{- 0 . 0 6} & \mathbf{- 0 . 0 6} & \mathbf{- 0 . 0 6} & -0.07 & -0.07 & -0.08 & 0.99 & -0.07 \\
-0.05 & -0.05 & -0.06 & -0.06 & -0.06 & -0.07 & -0.07 & -0.07 & 1.00
\end{array}\right],} \\
& K=-3.83 e-02 \times \\
& {\left[\begin{array}{lllllllll}
1.00 & 0.65 & 0.46 & 0.35 & 0.27 & 0.22 & 0.19 & 0.17 & 0.17 \\
0.65 & 0.93 & 0.60 & 0.41 & 0.31 & \mathbf{0 . 2 4} & \mathbf{0 . 2 0} & \mathbf{0 . 1 8} & 0.17 \\
0.46 & 0.60 & 0.89 & 0.56 & 0.39 & \mathbf{0 . 2 9} & \mathbf{0 . 2 3} & \mathbf{0 . 2 0} & 0.19 \\
0.35 & 0.41 & 0.56 & 0.87 & 0.55 & \mathbf{0 . 3 8} & \mathbf{0 . 2 9} & \mathbf{0 . 2 4} & 0.22 \\
0.27 & 0.31 & 0.39 & 0.55 & 0.86 & 0.55 & 0.39 & 0.31 & 0.27 \\
0.22 & \mathbf{0 . 2 4} & \mathbf{0 . 2 9} & \mathbf{0 . 3 8} & 0.55 & 0.87 & 0.56 & 0.41 & 0.35 \\
0.19 & \mathbf{0 . 2 0} & \mathbf{0 . 2 3} & \mathbf{0 . 2 9} & 0.39 & 0.56 & 0.89 & 0.60 & 0.46 \\
0.17 & \mathbf{0 . 1 8} & \mathbf{0 . 2 0} & \mathbf{0 . 2 4} & 0.31 & 0.41 & 0.60 & 0.93 & 0.65 \\
0.17 & 0.17 & 0.19 & 0.22 & 0.27 & 0.35 & 0.46 & 0.65 & 1.00
\end{array}\right]}
\end{aligned}
$$

It is evident that the coupling between the two group of signals separated by the central shield, which are shown in bold- 
face, is quite significant. If the shield width is increased to $1.5 \mu \mathrm{m}$, the matrices become

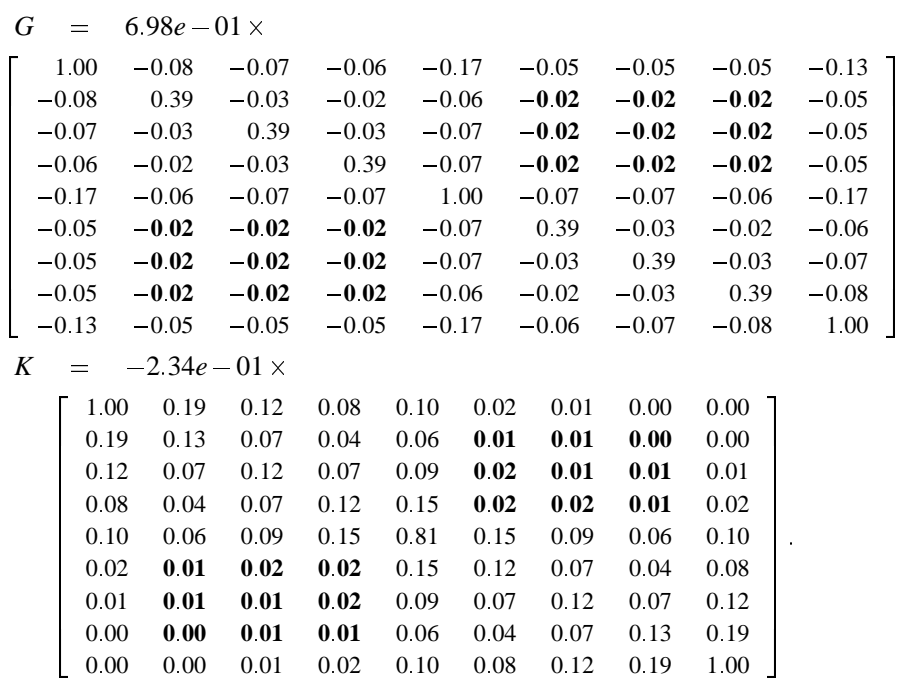

Now, the coupling between the two groups becomes much weaker, although the coupling within one group of signals is still quite significant.

Usually long signal busses should be divided into many segments in order to capture the distributive nature of interconnects. In the above example, we consider only one group of aligned segments in a bus structure for clarity of presentation. If all the segments are considered together, we can still obtain similar results and draw the same conclusion.

\section{VALIDATION}

In this section, SPICE simulations are carried out to validate the matrix $(R+j \omega L)^{-1}$ as a metric for effective inductive coupling. We consider a fully shielded structure with 15 signals and 16 shields. In this structure, odd wires are shields and even wires are signals. We consider two cases: First, we consider the case where the shields are perfect (zero resistance) and $L^{-1}$ is tridiagonal. We refer to it as the ideal case. Second, we consider the case where RLC parasitics are extracted from real circuits and refer to it as the realistic case.

In the bus structure, all the signal wires are $2 \mathrm{~mm}$ long and $1 \mu \mathrm{m}$ thick, representing typical top level interconnects. To capture the distributive nature of interconnects, we divide each wire into many segments along the length. PEEC model is used in the simulations. All the drivers are $160 \times$ of the minimum inverter in a representative $0.18 \mu \mathrm{m}$ CMOS technology with $1.5 \mathrm{~V} V_{d d}$, and the receivers are $40 \times$ of the minimum inverter. The input signal frequency is $2 \mathrm{GHz}$. The ramp is $1 / 10$ of the signal period.
The central signal (the 8th signal) is set to be the victim net as it is most strongly coupled to all other signal nets. To obtain the maximum noise, we keep the victim signal quiet and switch all other signals in the same direction. As the signal nets are not capacitively coupled to each other in a fully shielded structure, it is the inductive coupling between signals that contributes to the noise in the victim net.

\section{A. The Ideal Case}

In the preceding section, we show that when shield resistances are zero and $L^{-1}$ is tridiagonal, the effective inductive coupling between signals is zero. It means that the signals in such an ideal fully shielded structure will not affect each other and there is no inductive noise at all.

We set up such an ideal circuit from a real circuit: The original $L^{-1}$ matrix is truncated to make it tridiagonal, and then inverted back to get a new $L$ matrix. The new $L$ matrix is used in the HSPICE simulation.

The simulation results show that coupling noise is $1.2 \times$ $10^{-6} \mathrm{~V}$, which are purely due to numerical errors.

Although this is not a practical example, it is very interesting: there are mutual inductances between all the signals, but there is no effective inductive coupling! One might be tempted to conclude that the zero effective inductive coupling is already suggested by the tridiagonal property of $L^{-1}$. However, in this example, if we use realistic resistances for shields instead, SPICE shows a noise of $0.11 \mathrm{~V}$. In other words, the $L^{-1}$ matrix does not reflect the effective inductive coupling but the $(R+j \omega L)^{-1}$ matrix does.

\section{B. The Realistic Case}

In this case, we extract the RLC parasitics from realistic circuits. We study the effects of shield width on signal noise by varying the shield widths while keeping the spacing and signal width fixed at $0.5 \mu \mathrm{m}$. Four shield widths are considered: $0.5 \mu \mathrm{m}, 1.0 \mu \mathrm{m}, 1.5 \mu \mathrm{m}$, and $2.0 \mu \mathrm{m}$. We define the pitch of the fully shielded structure as the center-tocenter distance of two neighboring signals. That is, Pitch $=$ Width $_{\text {signal }}+$ Width $_{\text {shield }}+2 *$ Separation. The curve labeled " "Wide Shield", in Figure 3 corresponds to the maximum noise levels that the victim signal experiences at different shield widths. In that figure, the maximum noise levels are plotted at different pitches. As expected, the maximum noise level decreases from $0.28 \mathrm{~V}$ to $0.06 \mathrm{~V}$ as the shield width increases.

Next, we consider the effect of separation on effective inductive coupling. Metric $(R+j \omega L)^{-1}$ shows that the separation 


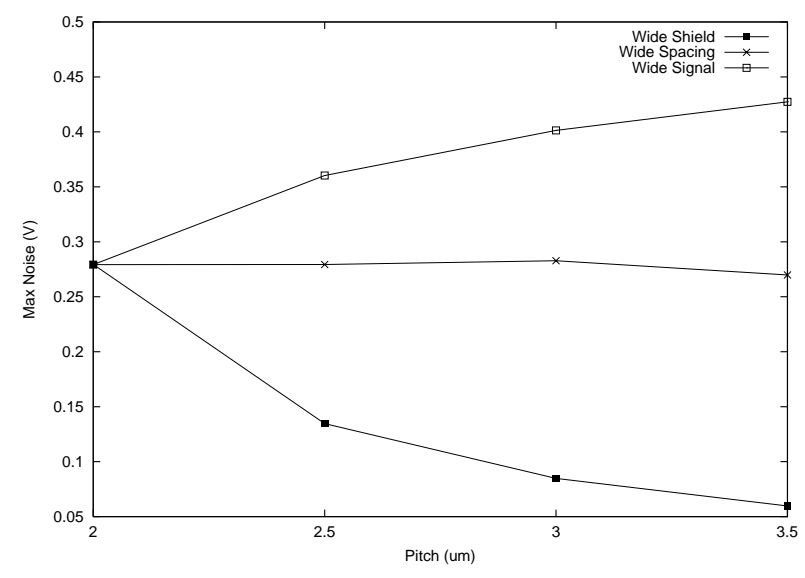

Fig. 3. Maximum noise levels at different pitches.

has little effect. To validate that, we consider a bus structure that is similar to the previous one but with fixed shield width $(0.5 \mu \mathrm{m})$. The signal width is also fixed at $0.5 \mu \mathrm{m}$. Here, we use four separations between wires such that the pitches match those of the previous bus structure. The curves labeled " Wide Spacing', 'in Figure 3 shows the maximum noise levels of the victim signal at different wire separations. We can observe that the maximum noise level reduces little as separation increases.

In the preceding section, the $G$ and $K$ matrices show that if the signal resistance is relatively lower than that of the shields, the effective inductive coupling between the signals will be strong. Another set of SPICE simulations is carried out to verify that. The shield width and wire separation are fixed at $0.5 \mu \mathrm{m}$, while three signal widths are considered: $0.5 \mu \mathrm{m}$, $1.0 \mu \mathrm{m}$, and $1.5 \mu \mathrm{m}$. In other words, the signal pitches used in this experiment match those in the earlier two experiments. The results are shown in the curves labeled " Wide Signal', in Figure 3. As expected, the noise increases as the signal width increases.

These SPICE simulations validate that $(R+j \omega L)^{-1}$ is a useful metric for effective inductive coupling.

\section{CONCLUSION}

In this paper, we study the effective inductive coupling problem. We propose to use matrix $(R+j \omega L)^{-1}$ as a metric for effective inductive coupling. We use this metric to analyze the effective inductive coupling of shielded structure and show how the resistances of the shields plays a role in the effectiveness of the shields. HSPICE simulations validate these analysis.

\section{REFERENCES}

[1] M. Beattie and L. Pileggi. Modeling magnetic coupling for on-chip interconnect. In Proc. Design Automation Conf, pages 335-340, 2001.

[2] A. Devgan, H. Ji, and W. Dai. How to efficiently capture on-chip inductance effects: introducing a new circuit element K. In Proc. Int. Conf. on Computer Aided Design, pages 150-155, 2000.

[3] K. Gala, V. Zolotov, R. Panda, B. Young, J. Wang, and D. Blaauw. On-chip inductance modeling and analysis. In Proc. Design Automation Conf, pages 63-68, 2000.

[4] R. M. Averill III and et al. Chip integration methodology for the IBM S/390 G5 and G6 custom microprocessors. IBM Journal of Research and Development, 43(5/6):681706, September/November 1999.

[5] S. Khatri, A. Mehrotra, R. Brayton, A. SangiovanniVincentelli, and R. Otten. A novel VLSI layout fabric for deep sub-micron applications. In Proc. Design Automation Conf, pages 491-496, 1999.

[6] Rajesh Kumar. Interconnect and noise immunity design for the Pentium 4 processor. Intel Technology Journal, Q1 2001.

[7] S. Lin, N. Chang, and S. Nakagawa. Quick on-chip self- and mutual-inductance screen. In IEEE International Symposium on Quality Electronic Design, pages 513-520, 2000.

[8] P. Restle, A. Ruehli, and S. Walker. Dealing with inductance in high-speed chip design. In Proc. Design Automation Conf, pages 904-909, 1999.

[9] A. E. Ruehli. Inductance calculation in a complex integrated circuit environment. IBM Journal of Research and Development, pages 470-481, September 1972. 